\title{
Explodability fluctuations of massive stellar cores enable asymmetric compact object mergers such as GW190814
}

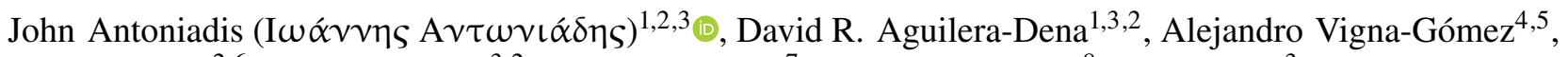 \\ Michael Kramer ${ }^{2,6}$, Norbert Langer ${ }^{3,2}$, Bernhard Müller ${ }^{7}$, Thomas M. Tauris ${ }^{8}$, Chen Wang ${ }^{3}$, and Xiao-Tian Xu ${ }^{3}$ \\ ${ }^{1}$ Institute of Astrophysics, Foundation for Research and Technology-Hellas, N. Plastira 100, Voutes 71003, Greece \\ e-mail: john@ia.forth.gr \\ 2 Max-Planck-Institut für Radioastronomie, Auf dem Hügel 69, 53121 Bonn, Germany \\ 3 Argelander-Institut für Astronomie, Universität Bonn, Auf dem Hügel 71, 53121 Bonn, Germany \\ 4 DARK, Niels Bohr Institute, University of Copenhagen, Jagtvej 128, 2200 Copenhagen, Denmark \\ 5 Niels Bohr International Academy, The Niels Bohr Institute, Blegdamsvej 17, 2100 Copenhagen, Denmark \\ 6 Jodrell Bank Centre for Astrophysics, University of Manchester, Manchester M13 9PL, UK \\ 7 School of Physics and Astronomy, Monash University, Clayton, VIC 3800, Australia \\ 8 Department of Physics and Astronomy, Aarhus University, Ny Munkegade 120, 8000 Aarhus, Denmark
}

Received 28 September 2021 / Accepted 1 December 2021

\begin{abstract}
The first three observing runs with Advanced LIGO and Virgo have resulted in the detection of binary black hole (BBH) mergers with highly unequal mass components, which are difficult to reconcile with standard formation paradigms. The most representative of these is GW190814, a highly asymmetric merger between a $23 M_{\odot}$ black hole $(\mathrm{BH})$ and a $2.6 M_{\odot}$ compact object. Here, we explore recent results, suggesting that a sizable fraction of stars with pre-collapse carbon-oxygen core masses above $10 M_{\odot}$, and extending up to at least $30 M_{\odot}$, may produce objects inside the so-called lower mass gap that bridges the division between massive pulsars and BHs in Galactic X-ray binaries. We demonstrate that such an explosion landscape would naturally cause a fraction of massive binaries to produce GW190814-like systems instead of symmetric-mass BBHs. We present examples of specific evolutionary channels leading to the formation of GW190814 and GW200210, a $24+2.8 M_{\odot}$ merger discovered during the O3b observing run. We estimate the merger-rate density of these events in our scenario to be $O(5 \%)$ of the total $\mathrm{BBH}$ merger rate. Finally, we discuss the broader implications of this formation channel for compact object populations, and its possible relevance to less asymmetric merger events such as GW200105 and GW200115.
\end{abstract}

Key words. gravitational waves - stars: massive - stars: black holes - stars: neutron - binaries: close - supernovae: general

\section{Introduction}

Following the detection of GW150914 - the first binary black hole $(\mathrm{BBH})$ merger observed in gravitational waves (GWs; Abbott et al. 2016a) - the three Advanced LIGO-Virgo Collaboration (henceforth LVC) observing runs (O1-3; Aasi et al. 2015; Acernese et al. 2015) have uncovered a diverse collection of GW signals originating in collisions between black holes (BHs) and neutron stars (NSs; Abbott et al. 2016b,c, 2017b, 2021b).

GW190814, discovered during O3 (Abbott et al. 2020), is an atypical event whose origin is particularly challenging to explain. Assuming no magnification due to gravitational lensing took place along the line-of-sight (Broadhurst et al. 2020), the signal corresponds to a merger between a $(23 \pm 1) M_{\odot}$ and a $(2.6 \pm 0.1) M_{\odot}$ object. While the massive primary is undoubtedly a $\mathrm{BH}$, the nature of the secondary is uncertain and not constrained by the GW signal itself: its mass securely places it inside the observed 'lower mass gap' (LMG) that separates the most massive NSs in binary pulsar systems $\left(\lesssim 2.1 M_{\odot}\right.$; Özel et al. 2012; Antoniadis et al. 2013, 2016; Fonseca et al. 2021) from the lightest BHs in X-ray binaries (XRBs; $\gtrsim 5 M_{\odot}$; Özel et al. 2010; Farr et al. 2011). If this object is a NS of $\sim 2.6 M_{\odot}$, its high mass, combined with previous constraints on NS properties (Özel \& Freire 2016; Abbott et al. 2017a) would place very stringent constraints on the behaviour of matter at extreme densities (for detailed discussions see e.g., Tsokaros et al. 2020; Nathanail et al. 2021). The possibility of a light $\mathrm{BH}$ instead is equally intriguing and forces us to rethink how such objects might form. More recently, the LVC announced the GWTC-3 catalogue which contains additional asymmetric systems (Abbott et al. 2021b). One event that stands out due to its similarity to GW190814 is GW200210, a 24 + $2.8 M_{\odot}$ compact-object merger. Even though this was a relatively low-significance detection (false-alarm-rate $>1 \mathrm{yr}^{-1}$ ), its discovery suggests that such asymmetric systems may be relatively common.

The combination of an extreme mass ratio and odd secondary mass in these systems poses an additional challenge for standard binary and dynamical formation channels. While certain scenarios are able to produce GW190814-like systems, they are either not common enough to match the empirically inferred mergerrate density of $1-23 \mathrm{Gpc}^{-3} \mathrm{yr}^{-1}$, or incompatible with the birth rates of other astrophysical populations (Kruckow et al. 2018; Zevin et al. 2020; Mandel et al. 2021, see Sect. 2). 
Here, we revisit isolated binary evolution channels in light of recent results suggesting that the landscape of core-collapse supernovae (CCSNe) may be substantially more complex than those predicted by standard binary population synthesis (BPS) prescriptions (Kruckow et al. 2018; Zevin et al. 2020; Mandel et al. 2021). The text is organised as follows: Sect. 2 reviews the properties of GW190814, as well as the proposed binary formation channels and their bottlenecks. In Sect. 3, we investigate the potential link between GW190814 and CCSNe originating from massive stars that would normally be expected to form highmass BHs. In Sect. 4, we present examples of specific formation channels and explore their implications for the merger-rate density of GW190814-like systems. We conclude with a summary in Sect. 5.

\section{Implications of GW190814-like mergers for binary evolution}

With a mass ratio of $q \simeq 0.1$, GW190814 and GW200210 are the most asymmetric compact binary mergers discovered to date. The observed GW190814 signal provided stringent constraints on the effective spin parameter, $\left|\chi_{\text {eff }}\right| \lesssim 0.063$, the magnitude of spin precession, $\chi_{\mathrm{p}} \leq 0.08$, and the spin magnitude of the more massive component, $\chi_{1} \leq 0.06$ (see Abbott et al. 2020, for details). Taken together, these estimates suggest that the primary was slowly rotating with a spin nearly aligned with the orbital angular momentum. These spin properties deviate from the general trend that has been found for less asymmetric systems, in which there seems to be a negative correlation between $q$ and $\chi_{\text {eff }}$, with more asymmetric systems having higher effective spins (Callister et al. 2021).

Assembly via dynamical channels, for instance in a nonsegregated cluster (Clausen et al. 2014; Fragione \& Banerjee 2020; Rastello et al. 2020), hierarchical mergers in multiple systems (Liu \& Lai 2021; Lu et al. 2021), and binary mergers near galactic nuclei (McKernan et al. 2020; Yang et al. 2020) may provide a viable formation pathway. However, these generally struggle to produce such highly asymmetric binaries at sufficient rates (see Abbott et al. 2020).

Isolated binary evolution channels producing GW190814like mergers have been extensively investigated by Zevin et al. (2020), who explored a broad range of physical assumptions using BPS tools (see also Mandel et al. 2021; Patton \& Sukhbold 2021; Shao \& Li 2021). Such systems might be produced via two broad binary formation channels: one in which the massive component forms first (henceforth Channel A) and another in which it forms last (Channel B). For Channel A, Zevin et al. (2020) identify the representative progenitor system to be a binary consisting of a $\sim 30 M_{\odot}$ zero-age main-sequence (ZAMS) star orbiting a $21 M_{\odot}$ star $(q \simeq 0.65)$ in a wide, $\sim 5000$-day, $e \simeq 0.3$ orbit. The primary loses mass via stable, non-conservative Case B mass transfer (Yoon et al. 2010; Tauris \& van den Heuvel 2022) that results in a moderate shrinkage of the orbit. The binary then receives a strong natal kick during the formation of the massive $\mathrm{BH}$ (post-collapse eccentricity of $e=0.98$ ). This is followed by a second episode of stable mass transfer onto the $\mathrm{BH}$, and a supernova $(\mathrm{SN})$ that forms the least-massive component and forces the binary into a more compact configuration via a second natal kick $(e=0.99)$. Overall, the combination of two explosions, both leading to highly eccentric post-SN configurations, is statistically quite rare.

In Channel $\mathrm{B}$, the typical progenitor is found to be a more compact binary consisting of a $\sim 45 M_{\odot}$ and a $\sim 23 M_{\odot}$ ZAMS star in a 2-day orbit. The system experiences two mass-transfer episodes that result in a mass inversion prior to the formation of the first compact object. The binary is kicked into a wider orbit during the SN (post-SN of $e=0.97$ ) and subsequently experiences a common envelope (CE) episode before the formation of the more-massive $\mathrm{BH}$. A similar channel has been identified by Mandel et al. (2021), who conclude that this path is only possible when Hertzsprung-gap donors are optimistically assumed to survive CE evolution (Klencki et al. 2021).

Zevin et al. (2020) find that at low metallicities, both pathways operate at nearly equal rates, while at higher metallicities, Channel A dominates. Their estimates depend on the CE efficiency, and the relation between ZAMS and compact-object masses. Matching the empirical GW190814-like merger-rate density is extremely challenging, regardless of the underlying assumptions.

In the context of the Fryer et al. (2012) SN prescription employed in Zevin et al. (2020), the mass of the secondary can only be explained via a delayed CCSN mechanism in which instabilities grow over timescales $\gtrsim 200 \mathrm{~ms}$, thereby allowing for more matter to be accreted onto the proto-NS compared to explosions in which such instabilities develop rapidly ( $\$ 50 \mathrm{~ms})$. While this naturally populates the LMG in all astrophysical populations, the initial binary configurations and required interactions that lead to the formation of highly asymmetric pairs are still highly improbable. The main reason is that LMG objects are only relevant to progenitors within a narrow-mass range, with pre-collapse carbon-oxygen $(\mathrm{CO})$ core masses $\left(M_{\mathrm{CO}}\right)$ of approximately $\sim 4 M_{\odot}$ (see Fryer et al. 2012, and Fig. 1). Such stars have a very small a priori probability of surviving the $\mathrm{SNe}$ and $\mathrm{CE}$ episodes required to form asymmetric compact binaries (e.g., Kruckow et al. 2016). In the following section, we explore how a physically motivated initial-to-remnant mass relation might help overcome this bottleneck.

\section{The birth mass distribution of compact objects}

As discussed in Sect. 2, rate estimates for GW190814-like mergers in BPS studies are severely limited by the range of stars that can produce compact objects inside the LMG. Consequently, a modified explosion landscape that increases the diversity of progenitors for the low-mass component compared to standard prescriptions (e.g., Hurley et al. 2000; Fryer et al. 2012) may significantly alter this picture.

Indeed, several recent studies suggest that the initialto-remnant mass relations traditionally employed in BPS simulations might not be representative of the true explosion landscape (O'Connor \& Ott 2011; Ugliano et al. 2012; Sukhbold \& Woosley 2014; Pejcha \& Thompson 2015; Ertl et al. 2016, 2020; Müller et al. 2016; Ebinger et al. 2019; Woosley 2019; Schneider et al. 2021; Patton \& Sukhbold 2020, 2021; Mandel et al. 2021; Shao \& Li 2021; Zapartas et al. 2021; Laplace et al. 2021; Aguilera-Dena et al. 2022). Whether a star forms a NS or a $\mathrm{BH}$ within the neutrino-driven explosion paradigm is largely determined by the pre-SN density (or equivalently, the 'compactness'; O'Connor \& Ott 2011) of the stellar core. In turn, the latter appears to depend sensitively on how the late stages of nuclear burning proceed, both inside the core and in the envelope (e.g., Brown et al. 2001; Yoshida et al. 2021). O'Connor \& Ott (2011), Ugliano et al. (2012), and Sukhbold \& Woosley (2014), employing simple explodability criteria, first showed that the complex processes taking place during these late evolutionary stages can lead to rapid alternations between successful explosions and implosions within certain mass ranges. More recently, it has been shown that 'islands of explodability' might 


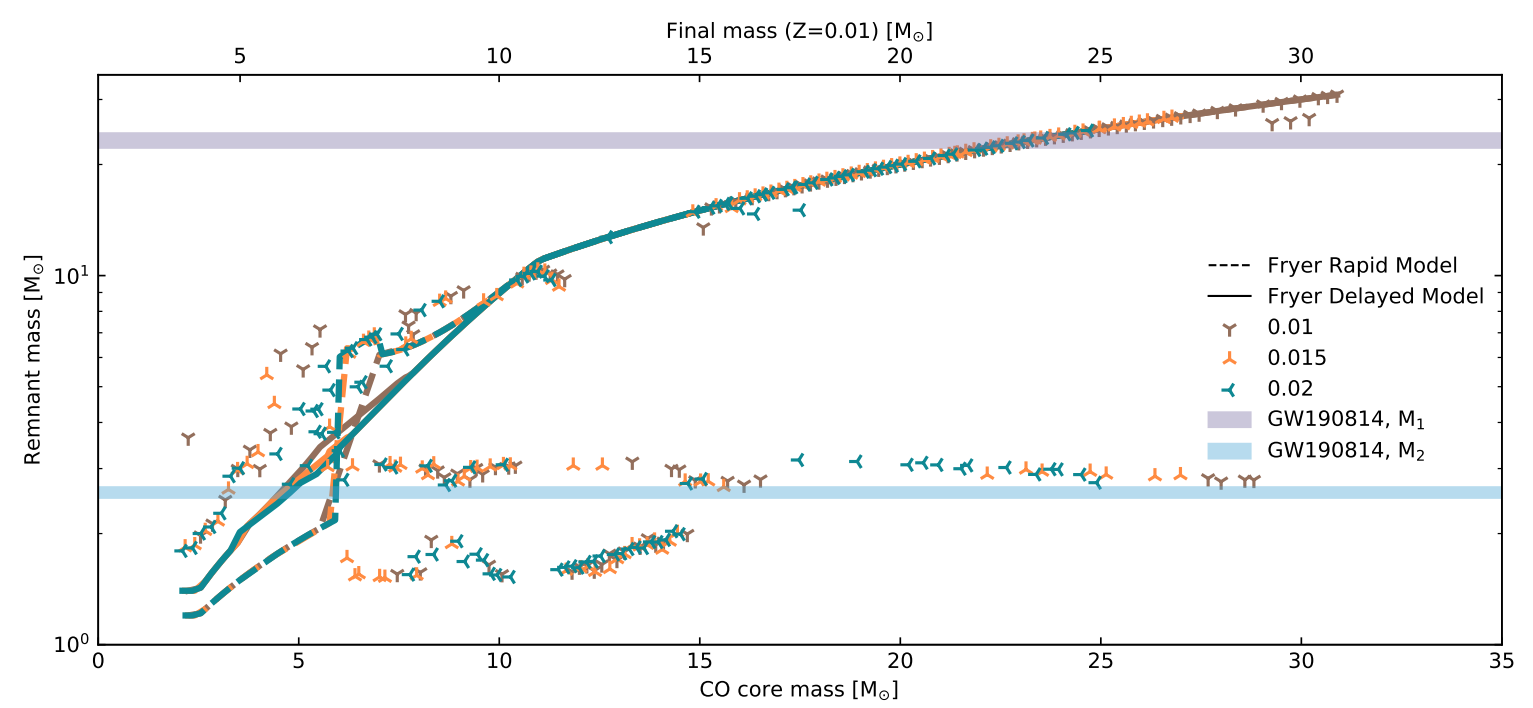

Fig. 1. Relation between remnant, CO-core, and pre-collapse masses derived from the helium-star models of Aguilera-Dena et al. (2022) using the semi-analytic explosion models of Müller et al. (2016) as updated in Mandel \& Müller (2020). The scatter plot illustrates the predictions of the aforementioned models for different initial metallicities $(Z=0.01,0.015,0.02$ shown in brown, orange, and green, respectively). Lines indicate the predictions of Fryer et al. (2012) and their variation with metallicity. Horizontal strips show the inferred component masses of GW190814. As can be seen, detailed models predict a much broader rage of possible progenitors for the low-mass component compared to standard BPS prescriptions. Notably, two CO cores of equal mass $\left(\sim 24 M_{\odot}\right)$ may reproduce both GW190814 components.

be even more pronounced in stars that are stripped of their hydrogen envelopes, for example due to binary interactions (Ertl et al. 2020; Schneider et al. 2021; Mandel et al. 2021; Laplace et al. 2021).

Recently, Aguilera-Dena et al. (2022, henceforth AD21; see also Aguilera-Dena et al. 2021) demonstrated, for the first time, that such pre-SN compactness variations extend to precollapse CO-core masses significantly above $10 M_{\odot}$, suggesting that successful CCSN explosions might occur even for the most massive stars. Figure 1 illustrates the correlations between remnant, $\mathrm{CO}-$ core, and pre-SN inferred by $\mathrm{AD} 21$. These relations are based on 1D evolutionary models of helium stars, calculated at different metallicities using the MESA code (Paxton et al. 2011, 2019). Remnant masses were inferred using a revised version of the Müller et al. (2016) parametric explosion models (see Mandel \& Müller 2020), which considers the effect of SN fallback. These semi-analytic prescriptions are motivated by 3D neutrino-driven simulations and rely on the pre-SN stellar structure to predict explosion properties and remnant masses (for a more detailed discussion on the model and the input parameters used to infer the quantities in Fig. 1, see AD21). As can be seen, the predictions of AD21 differ substantially from analytic BPS prescriptions (e.g., Hurley et al. 2000; Fryer et al. 2012). Contrary to the latter, both NSs and LMG objects are obtained for stars with a final $M_{\mathrm{CO}}$ extending up to $\sim 30 M_{\odot}$. The mass range for which explosions occur appears to depend on metallicity; for example, for $Z=0.02$, one obtains explosions for $M_{\mathrm{CO}} \leq 25 M_{\odot}$, while for $Z=0.01$ one finds explosions extending to $M_{\mathrm{CO}} \leq 30 M_{\odot}$. Most notably, our models suggest the presence of an extended and well-defined explodability island in the $M_{\mathrm{CO}}=10 \ldots 15 M_{\odot}$ range (first identified by Schneider et al. 2021), where CCSNe (leading to NS formation) are significantly favoured over implosions (leaving behind BHs). For $M_{\mathrm{CO}} \geq 15 M_{\odot}$, while the most likely outcome is an implosion, we predict that a sizable fraction of stars $(10 \%$ and $20 \%$ of our models at $Z=0.01$ and 0.02 , respectively) produce a fallback CCSN that leads to the formation of a LMG object, instead of a massive $\mathrm{BH}$. These remnants have masses between
2.6 and $3.1 M_{\odot}$, i.e. quite similar to the low-mass component in GW190814. Here, for simplicity, we assume all LMG objects to be BHs, given the current constraints on the equation-of-state. This choice affects our results only moderately, as one would expect somewhat higher kick velocities (up to $\sim 1200 \mathrm{~km} \mathrm{~s}^{-1}$ ) if the remnant is a NS.

The aforementioned properties make high-mass stars particularly appealing as GW190814 progenitors for two reasons. Firstly, due to underlying dependence on metallicity and the shape of the initial mass function (IMF; Kroupa 2001), they are expected to be rare, and hence not particularly relevant to the LMG in Galactic populations. Secondly, despite their rarity they are very similar to the stars expected to form the more massive component. Such progenitors are extremely similar to those expected to form more 'canonical' symmetric binary BHs (Abbott et al. 2021a). Hence, they have increased chances of producing a successful merger (e.g., Belczynski et al. 2016; Kruckow et al. 2018; Vigna-Gómez et al. 2018) compared to the systems identified by Zevin et al. (2020) and Mandel et al. (2021). In the following section, we give specific examples of symmetric binaries that may lead to the formation of GW190814 based on the explosion landscape discussed here.

\section{Binary formation channels for GW190814}

In this section, we demonstrate the impact of a non-monotonic relation between pre-SN and remnant masses on the formation of highly asymmetric mergers, using two specific evolutionary examples. While both paradigms are subject to several physical uncertainties (e.g., related to the modelling of Case A mass transfer in BPS codes, or the outcome of CE events), they serve to demonstrate that GW190814-like systems and symmetric BBHs $(q \simeq 1)$ may form in a very similar way.

Figure 2 summarises the evolution of these example systems, assuming the AD21 explosion landscape discussed in Sect. 4 and Fig. 1. The left-hand side shows a Channel A-type progenitor (see also Fig. A.1) that was calculated using COMPAS 
(Team COMPAS 2021). By employing this rapid BPS code, we were able to rapidly identify this particular example among several models, using methods and assumptions similar to those adopted by previous population-synthesis studies (e.g., Zevin et al. 2020).

The initial system has a very low metallicity (1\% solar) and consists of a $55.2+32.5 M_{\odot}$ ZAMS pair, separated by $0.6 \mathrm{AU}$. The system undergoes stable Case A mass transfer, which is followed by the formation of a $21.4 M_{\odot} \mathrm{BH}$ from the initially more massive star. The secondary then expands during core helium burning $(\mathrm{CHeB})$. This leads to a $\mathrm{CE}$ episode that expels the envelope and hardens the binary. The resulting system evolves without experiencing further mass transfer, until the secondary collapses. Based on the default Fryer et al. (2012) remnantmass prescription employed in COMPAS, the resulting system would be a 'canonical' $21.4+19.5 M_{\odot}$ binary BH system. Such symmetric $\mathrm{BBH}$ mergers are expected to be the most common and may originate from a broad range of initial configurations (e.g., Belczynski et al. 2016; Stevenson et al. 2017), in addition to the one discussed here. However, as shown in Sect. 3, the secondary might instead produce a LMG object in a fallback CCSN at the 10-20\% probability level, thereby creating a GW190814like system.

Even though this demonstrates that symmetric- and asymmetric-mass mergers could have nearly identical formation histories, one key difference that can set them apart is the magnitude of the natal kick received during the CCSN. Our models predict a wide range of kick velocities for fallback CCSNe extending from 0 to $\sim 1200 \mathrm{~km} \mathrm{~s}^{-1}$. This diversity is an intentional feature of our semi-analytic SN prescription that is physically motivated by the results of detailed 3D simulations (see Mandel \& Müller 2020; Chan et al. 2020, and AD21 for details). For stellar structures that yield low initial explosion energies, one expects the asymmetric inner ejecta to fall back completely. A low-energy explosion then results from a weak sound pulse that is launched when the initial ejecta become subsonic. The transition to the subsonic regime means that the transport of energy by the sound pulse decouples from the transport of matter (with the initial ejecta being accreted onto the black hole). The sound pulse quickly becomes spherical resulting in symmetric explosions with negligible natal kicks. Alternatively, if the initial ejecta has sufficiently high energy, it escapes and the momentum asymmetry between the ejecta and the remnant is largely preserved, resulting in a strong kick.

Evidently, symmetric explosions would not significantly affect the binary dynamics, as they may only modify the eccentricity by an amount proportional to the mass lost in the explosion ( $e=\delta M / M \simeq 0.7$ for the example in Fig. 2, where $\delta M$ is the ejected mass, and $e$ and $M$ are the post-SN eccentricity and total mass of the binary, respectively). As long as $\delta M$ is smaller than the total mass, the system remains bound and merges on a similar timescale compared to circular systems with comparable separations. On the other hand, CCSNe that produce large kicks may completely alter the binary dynamics (see Tauris et al. 2017, and references therein). Assuming the largest kick velocity obtained for our models $\left(w=1200 \mathrm{~km} \mathrm{~s}^{-1}\right)$ and a random kick orientation, for the example in Fig. 2, one finds a 34\% probability of surviving and merging within a Hubble time (see also Fig. A.3). This estimate is likely extremely conservative, as nearly all fallback CCSNe in our calculations produce smaller kicks.

To summarise, given the similarities to channels that form symmetric-mass $\mathrm{BH}$ pairs, we believe Channel $\mathrm{A}$ to be the dominant contributor of GW190814-like systems, especially at low metallicities. A 0th-order semi-empirical esti-

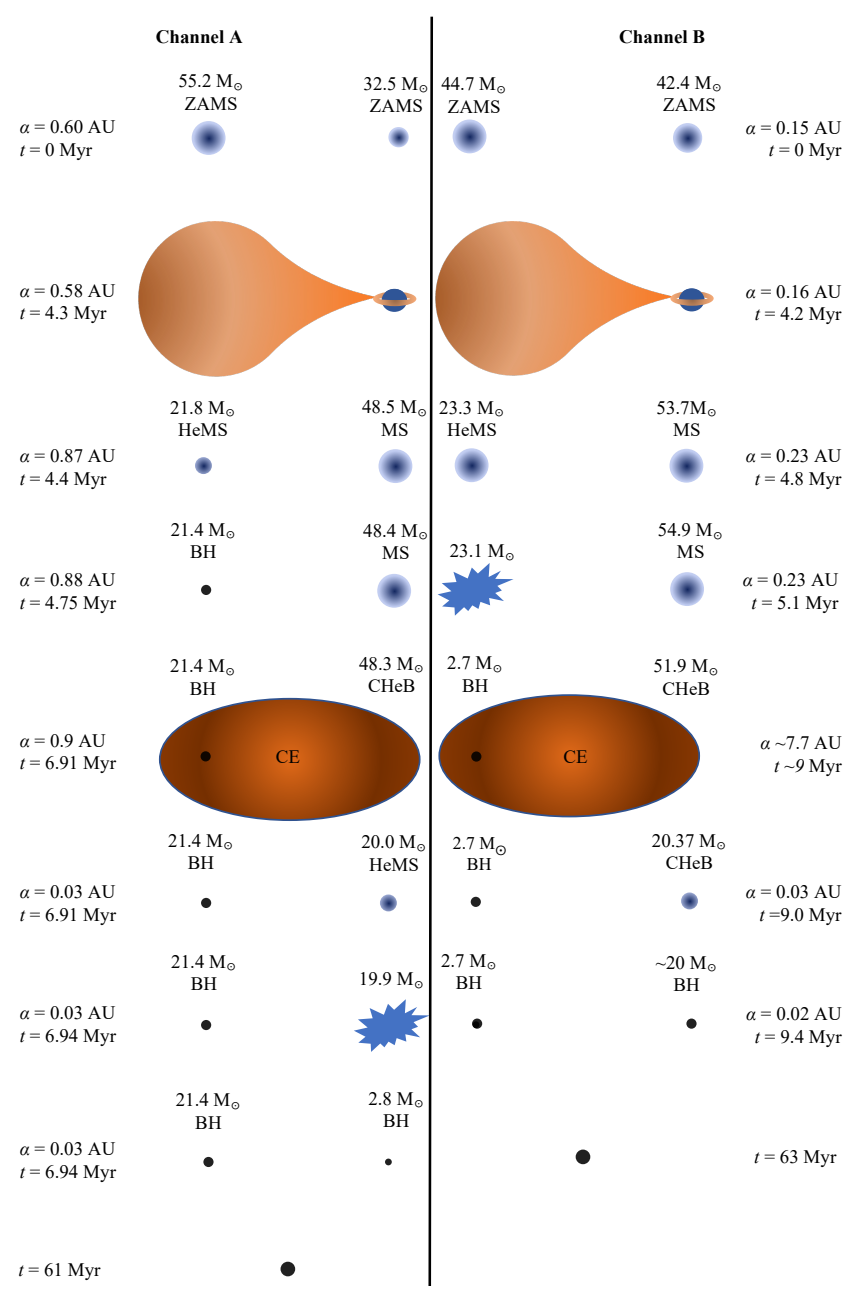

Fig. 2. Examples of binary evolution leading to the formation of asymmetric-mass GW190814-like mergers.

mate for the expected rates under our scenario can be obtained from the observed $\mathrm{BBH}$ merger rates. The LIGO Scientific Collaboration et al. (2021) estimate the total merger rate for BBHs to be $R_{\mathrm{BBH}}=16-130 \mathrm{Gpc}^{-3} \mathrm{yr}^{-1}$ (90\% credible interval). If the majority of GW190814-like systems form in a similar way to symmetric BBHs, then their expected rate would be the following:

$R_{\mathrm{GW} 190814} \simeq R_{\mathrm{BBH}} \times F_{\text {fallback }} \times P_{\text {survive }}$,

where $F_{\text {fallback }}$ is the IMF-weighted fraction of stars that produce fallback $\mathrm{SNe}$ instead of massive $\mathrm{BHs}$, and $P_{\text {survive }}$ is the population-averaged probability for such systems to survive the natal kick and produce a successful merger. Therefore, if $F_{\text {fallback }} \simeq O(10 \%)$, as suggested by our models, and $P_{\text {survive }} \simeq O(50 \%)$ similarly to the (conservative) example above, one obtains $R_{q \neq 1} \simeq 0.75-6.5 \mathrm{Gpc}^{-3} \mathrm{yr}^{-1}$, which is in good agreement with the empirical estimate in Abbott et al. (2020). A robust independent measurement of $R_{\mathrm{GW} 190814}$ in future observing runs with GW detectors, could serve as a direct proxy for $F_{\text {fallback }} \times P_{\text {survive }}$.

We now examine the possibility of the low-mass component forming first (Channel B). An example binary that follows this channel is shown on the right-hand side of Fig. 2 (see also Fig. A.2). The model is taken from the detailed binary evolution tracks of Wang et al. (2020) (see also Langer et al. 2020), calculated at the metallicity of the Small Magellanic Cloud (SMC). 
The initial binary consists of a 44.7 and a $42.4 M_{\odot}$ star in 5-day orbit. The system first undergoes Case A mass transfer from the primary to the secondary, which leads to a mass-ratio inversion. The CCSN that forms the LMG object occurs when the separation is $\sim 0.23 \mathrm{AU}$. The $\mathrm{AD} 21$ model that resembles the precollapse state of the progenitor best has $M_{\mathrm{final}}=23.5 M_{\odot}$ and a (higher) metallicity of $z=0.01$. This model can produce a $2.7 M_{\odot} \mathrm{BH}$ that receives a $\sim 400 \mathrm{~km} \mathrm{~s}^{-1} \mathrm{kick}$. For these parameters, the system has a $\sim 38 \%$ probability to remain bound without the LMG BH falling inside its companion at periastron, and a $\sim 1 \%$ chance of being kicked into a wide orbit ( $25 \mathrm{AU} ; e \gtrsim 0.8$ ), which would allow the secondary to further evolve without transferring mass until the late $\mathrm{CHeB}$ stages.

To better evaluate the final outcome of this evolutionary path, we used MESA to evolve a $2.7+54.9 M_{\odot} \mathrm{BH}$ and MS binary model, with an orbit identical to the post-SN Case B example of Zevin et al. (2020) $(a=9 \mathrm{AU} ; e=0.95)$ at the SMC metallicity $\left(Z=2.179 \times 10^{-3}\right.$; see Appendix). The binary initiates unstable mass transfer and enters a $\mathrm{CE}$ during $\mathrm{CHeB}$ (at $t=9 \mathrm{Myr}$ ), when $a=7.7 \mathrm{AU}, e=0.94$, and $m_{2}=51.9 M_{\odot}$. At this stage, the envelope of the secondary has a binding energy of $E_{\text {bind }}=5.8 \times 10^{49} \mathrm{erg}$ (evaluated when the Roche lobe is filled; $\left.R_{\mathrm{RL}}=1047 R_{\odot}\right)$. This means that a relatively high CE efficiency of $\alpha \equiv E_{\text {bind }} / \Delta E_{\text {orb }} \gtrsim 1.7$ (where $\Delta E_{\text {orb }} \simeq-G m_{1} m_{2}^{\text {final }} / 2 a^{\text {final }}$ is the orbital energy, see Kruckow et al. 2016) is required to successfully eject the envelope. For $\alpha \gtrsim 2.8$, the post-CE binary remains detached and produces a GW190814-like system with a $20 M_{\odot} \mathrm{BH}$ that merges within tens of millions of years. Alternatively, if the post-CE separation is tighter, the binary may experience further (unstable) mass transfer leading to the exposure of the secondary's $15 M_{\odot} \mathrm{CO}$ core. In this case the end-product could be either a less asymmetric BBH (with $q \simeq 0.18$ versus 0.12 ) or another LMG object or NS (in which case, the system would be disrupted).

Assuming that after the formation of the low-mass $\mathrm{BH}$, the evolution of the aforementioned system is similar to the Channel B example of Zevin et al. (2020), then to first order, the relative contribution of the two binary types to the observed merger-rate density is determined by the frequency of the respective ZAMS progenitors. Assuming a Kroupa (2001) IMF and the Sana et al. (2012) distributions for the initial binary parameters, then one would expect the merger-rate contribution of our Fig. 2 example to be comparable to that in Zevin et al. (2020). This is likely conservative, given the broad mass range over which LMG objects form in our models. Nevertheless, Channel B should be rare compared to Channel $\mathrm{A}$, mainly because it requires both a fine-tuned SN kick and a high CE efficiency. However, as already demonstrated by Zevin et al. (2020), it could play an increasingly important role at higher metallicities.

Nevertheless, Channel A also more naturally explains the spin properties of GW190814 as one may expect the massive $\mathrm{BH}$ to have a small spin that is likely aligned with the orbital angular momentum, thereby resulting in a small $\chi_{\text {eff }}$, consistent with what has been observed. While the second SN may lead to misalignment, the orbit at that time is quite compact, causing the magnitude of the kick velocity to be comparable to the pre-SN orbital velocity. In turn, this should cause the post-SN misalignment to be small (Tauris \& van den Heuvel 2022). Contrarily, Channel B would likely produce a fast-spinning massive $\mathrm{BH}$. While one would still expect the spin to be aligned with the orbital angular momentum, this might have produced some additional observable signature in the GW signal (i.e. $\chi_{1} \neq 0$; Qin et al. 2018). The small $\chi_{\text {eff }}$ in our scenario would also explain why the spin properties of GW190814 resemble those of BBH mergers with $q \sim 1$, and significantly deviate from the recently reported anti-correlation between $q$ and $\chi$ eff found for the general BBH population (derived for mergers with $q$ in the $0.4-1$ range and excluding GW190814; see Callister et al. 2021).

\section{Summary}

In this work we investigated the potential role of explodability islands in the formation of highly asymmetric-mass mergers such as GW190814 and the recently announced GW200210. We used a dense grid of SN progenitor models at different metallicities (presented in detail in Aguilera-Dena et al. 2022) to demonstrate that both NSs and LMG objects - which are assumed to be BHs here - may be produced from a broad range of massive stars, with $M_{\mathrm{CO}}$ masses extending up to $\sim 30 M_{\odot}$ (Sect. 3; Fig. 1). This picture agrees well with several recent studies (e.g., Laplace et al. 2021; Schneider et al. 2021, and references above), but it is in a stark contrast to standard BPS prescriptions, in which LMG objects may only form from an extremely limited range of progenitors with pre-collapse $M_{\mathrm{CO}} \simeq 4 M_{\odot}$. In Sect. 4, we demonstrate that a direct consequence of this modified explosion landscape is that asymmetric GW190814like mergers may form in nearly identical way to 'canonical' equal-mass BH mergers (e.g., Belczynski et al. 2016), the only difference being that the second core collapse event makes a LMG object instead of a high-mass BH. If this is indeed the case, then the merger rate of asymmetric systems that formed via this path should be a sizable fraction of the total $\mathrm{BBH}$ rate, proportional to the number of high-mass stars that fall inside explodability islands (Sect. 4; Eq. (1)). A major difference is that, on-average, fallback CCSNe are expected to yield sizable natal kicks. Combining our AD21 progenitor models with the semi-analytic core-collapse prescription of Mandel \& Müller (2020), we find that LMG objects may receive kicks ranging from 0 to $\sim 1200 \mathrm{~km} \mathrm{~s}^{-1}$. Our results for the highest magnitude kicks (Sect. 4, Fig. A.3) suggest that $O(50 \%)$ of systems evolving via this channel should be able to survive the $\mathrm{SN}$ explosion and produce a successful merger. These results motivate further BPS simulations with updated final-to-remnant mass relations and kick distributions. Such studies should be able to further constrain the merger-rate densities of asymmetric-mass mergers and the (likely negligible) consequences for the LMG in Galactic binaries. Our Channel B estimates suggest that this path is rare compared to Channel A. Nevertheless, it may play an important role in high-metallicity environments and may also be relevant to the formation of binary compact objects in the Galaxy (e.g., $\mathrm{BH}$ binaries with mildly recycled pulsar companions, or ultramassive X-ray binaries such as GX 301-2 Doroshenko et al. 2010). Similarly, NSs and LMG objects originating from stars with $M_{\mathrm{CO}} \geq 10 M_{\odot}$ may also contribute to mergers that are less asymmetric than GW190814 and GW200210. For instance, the $7 \lesssim M_{\mathrm{CO}} / M_{\odot} \lesssim 15$ regime, which may contribute objects with masses of $\sim 1.4,3$ and $\sim 8 M_{\odot}$ (Fig. 1), could be relevant to the first two NSBH mergers GW200115 and GW200105 (component masses of $\left(\sim 1.6+6\right.$ and $1.9+8.9 M_{\odot}$, respectively Vigna-Gómez et al. 2021; Broekgaarden \& Berger 2021). A more detailed exploration of the entire parameter space shown in Fig. 1 is necessary to investigate the full range of possible outcomes, as well as potential contradictions to observations.

As a final remark we note that fallback SNe leading to the formation of NSs or LMG objects in symmetric explosions, may also carry broader implications for compact-object populations. For instance, they might contribute to the observed pulsar population in globular clusters, thereby serving as a substitute for 
NSs that formed via electron-capture SNe, which may be rarer than previously thought (Antoniadis et al. 2020).

Acknowledgements. This work was supported by the Stavros Niarchos Foundation (SNF) and the Hellenic Foundation for Research and Innovation (H.F.R.I.) under the 2nd Call of "Science and Society" Action Always strive for excellence - "Theodoros Papazoglou" (Project Number: 01431). A.V.-G. acknowledges support by the Danish National Research Foundation (DNRF132). Simulations in this paper made use of the COMPAS rapid binary population synthesis code (version 02.22.00), which is freely available at http://github.com/ TeamCOMPAS/COMPAS

\section{References}

Aasi, J., Abbott, B. P., Abbott, R., et al. 2015, CQG, 32, 074001

Abbott, B. P., Abbott, R., Abbott, T. D., et al. 2016a, Phys. Rev. Lett., 116, 061102

Abbott, B. P., Abbott, R., Abbott, T. D., et al. 2016b, Phys. Rev. Lett., 116, 241103

Abbott, B. P., Abbott, R., Abbott, T. D., et al. 2016c, Phys. Rev. X, 6, 041015

Abbott, B. P., Abbott, R., Abbott, T. D., et al. 2017a, ApJ, 848, L12

Abbott, B. P., Abbott, R., Abbott, T. D., et al. 2017b, Phys. Rev. Lett., 119, 161101

Abbott, R., Abbott, T. D., Abraham, S., et al. 2020, ApJ, 896, L44

Abbott, R., Abbott, T. D., Acernese, F., et al. 2021a, GWTC-3: Compact Binary Coalescences Observed by LIGO and Virgo During the Second Part of the Third Observing Run

Abbott, R., Abbott, T. D., Abraham, S., et al. 2021b, ApJ, 913, L7

Acernese, F., Agathos, M., Agatsuma, K., et al. 2015, CQG, 32, 024001

Aguilera-Dena, D. R., Langer, N., Antoniadis, J., et al. 2021, A\&A, submitted [arXiv:2112.06948]

Aguilera-Dena, D. R., Langer, N., Antoniadis, J., et al. 2022, A\&A, submitted

Antoniadis, J., Chanlaridis, S., Gräfener, G., \& Langer, N. 2020, A\&A, 635, A72

Antoniadis, J., Freire, P. C. C., Wex, N., et al. 2013, Science, 340, 448

Antoniadis, J., Tauris, T. M., Özel, F., et al. 2016, ArXiv e-prints [arXiv:1605.01665]

Belczynski, K., Holz, D. E., Bulik, T., \& O’Shaughnessy, R. 2016, Nature, 534, 512

Broadhurst, T., Diego, J. M., \& Smoot, G. F. 2020, ArXiv e-prints [arXiv:2006. 13219]

Broekgaarden, F. S., \& Berger, E. 2021, ApJ, 920, L13

Brown, G. E., Heger, A., Langer, N., et al. 2001, New Astron., 6, 457

Callister, T. A., Haster, C.-J., Ng, K. K. Y., Vitale, S., \& Farr, W. M. 2021, ApJ, 922, L5

Chan, C., Müller, B., \& Heger, A. 2020, MNRAS, 495, 3751

Clausen, D., Sigurdsson, S., \& Chernoff, D. F. 2014, MNRAS, 442, 207

Doroshenko, V., Santangelo, A., Suleimanov, V., et al. 2010, A\&A, 515, A10

Ebinger, K., Curtis, S., Fröhlich, C., et al. 2019, ApJ, 870, 1

Ertl, T., Janka, H.-T., Woosley, S. E., Sukhbold, T., \& Ugliano, M. 2016, ApJ, 818,124

Ertl, T., Woosley, S. E., Sukhbold, T., \& Janka, H.-T. 2020, ApJ, 890, 51

Farr, W. M., Sravan, N., Cantrell, A., et al. 2011, ApJ, 741, 103L

Fonseca, E., Cromartie, H. T., Pennucci, T. T., et al. 2021, ApJ, 915, L12

Fragione, G., \& Banerjee, S. 2020, ApJ, 901, L16

Fryer, C. L., Belczynski, K., Wiktorowicz, G., et al. 2012, ApJ, 749, 91
Hills, J. G. 1983, ApJ, 267, 322

Hurley, J. R., Pols, O. R., \& Tout, C. A. 2000, MNRAS, 315, 543

Klencki, J., Nelemans, G., Istrate, A. G., \& Chruslinska, M. 2021, A\&A, 645, A54

Kroupa, P. 2001, MNRAS, 322, 231

Kruckow, M. U., Tauris, T. M., Langer, N., et al. 2016, A\&A, 596, A58

Kruckow, M. U., Tauris, T. M., Langer, N., Kramer, M., \& Izzard, R. G. 2018, MNRAS, 481, 1908

Langer, N., Schürmann, C., Stoll, K., et al. 2020, A\&A, 638, A39

Laplace, E., Justham, S., Renzo, M., et al. 2021, A\&A, 656, A58

Liu, B., \& Lai, D. 2021, MNRAS, 502, 2049

Lu, W., Beniamini, P., \& Bonnerot, C. 2021, MNRAS, 500, 1817

Mandel, I., \& Müller, B. 2020, MNRAS, 499, 3214

Mandel, I., Müller, B., Riley, J., et al. 2021, MNRAS, 500, 1380

McKernan, B., Ford, K. E. S., \& O’Shaughnessy, R. 2020, MNRAS, 498, 4088

Müller, B., Heger, A., Liptai, D., \& Cameron, J. B. 2016, MNRAS, 460, 742

Nathanail, A., Most, E. R., \& Rezzolla, L. 2021, ApJ, 908, L28

O'Connor, E., \& Ott, C. D. 2011, ApJ, 730, 70

Özel, F., \& Freire, P. 2016, ARA\&A, 54, 401

Özel, F., Psaltis, D., Narayan, R., \& McClintock, J. E. 2010, ApJ, 725, 1918

Özel, F., Psaltis, D., Narayan, R., \& Santos Villarreal, A. 2012, ApJ, 757, 55

Patton, R. A., \& Sukhbold, T. 2020, MNRAS, 499, 2803

Patton, R. A., Sukhbold, T., \& Eldridge, J. J. 2021, MNRAS, submitted [arXiv:2106.05978]

Paxton, B., Bildsten, L., Dotter, A., et al. 2011, ApJS, 192, 3

Paxton, B., Marchant, P., Schwab, J., et al. 2015, ApJS, 220, 15

Paxton, B., Smolec, R., Schwab, J., et al. 2019, ApJS, 243, 10

Pejcha, O., \& Thompson, T. A. 2015, ApJ, 801, 90

Peters, P. C. 1964, Phys. Rev., 136, 1224

Qin, Y., Fragos, T., Meynet, G., et al. 2018, A\&A, 616, A28

Rastello, S., Mapelli, M., Di Carlo, U. N., et al. 2020, MNRAS, 497, 1563

Sana, H., de Mink, S. E., de Koter, A., et al. 2012, Science, 337, 444

Schneider, F. R. N., Podsiadlowski, P., \& Müller, B. 2021, A\&A, 645, A5

Shao, Y., \& Li, X.-D. 2021, ApJ, 920, 81

Stevenson, S., Vigna-Gómez, A., Mandel, I., et al. 2017, Nat. Commun., 8, 14906 Sukhbold, T., \& Woosley, S. E. 2014, ApJ, 783, 10

Tauris, T. M., Kramer, M., Freire, P. C. C., et al. 2017, ApJ, 846, 170

Tauris, T. M., \& van den Heuvel, E. P. J. 2022, Physics of Binary Star Evolution (Princeton University Press)

Team COMPAS (Riley, J., et al.) 2021, ArXiv e-prints [arXiv:2109. 10352]

The LIGO Scientific Collaboration, the Virgo Collaboration, \& the KAGRA Collaboration (Abbott, R., et al.) 2021 ArXiv e-prints [arXiv:2111.03634]

Tsokaros, A., Ruiz, M., \& Shapiro, S. L. 2020, ApJ, 905, 48

Ugliano, M., Janka, H.-T., Marek, A., \& Arcones, A. 2012, ApJ, 757, 69

Vigna-Gómez, A., Neijssel, C. J., Stevenson, S., et al. 2018, MNRAS, 481, 4009

Vigna-Gómez, A., Schrøder, S. L., Ramirez-Ruiz, E., et al. 2021, ApJ, 920, L17

Vink, J. S. 2017, A\&A, 607, L8

Vink, J. S., de Koter, A., \& Lamers, H. J. G. L. M. 2001, A\&A, 369, 574

Wang, C., Langer, N., Schootemeijer, A., et al. 2020, ApJ, 888, L12

Woosley, S. E. 2019, ApJ, 878, 49

Yang, Y., Gayathri, V., Bartos, I., et al. 2020, ApJ, 901, L34

Yoon, S., Woosley, S. E., \& Langer, N. 2010, ApJ, 725, 940

Yoshida, T., Takiwaki, T., Aguilera-Dena, D. R., et al. 2021, MNRAS, 506, L20

Zahn, J.-P. 1977, A\&A, 500, 121

Zapartas, E., Renzo, M., Fragos, T., et al. 2021, A\&A, 656, L19

Zevin, M., Spera, M., Berry, C. P. L., \& Kalogera, V. 2020, ApJ, 899, L1 


\section{Appendix A: Supplementary material}

\section{MESA simulations}

Here, we briefly describe the set-up and input physics of our MESA simulations for the Channel B progenitor discussed in Section 4 . The initial system is a $44.7+42.7 M_{\odot}$ ZAMS pair with an SMC metallicity $\left(Z=2.179 \times 10^{-3}\right)$, separated by $0.15 \mathrm{AU}$. The evolution of the binary up to the formation of the first compact object was calculated by Wang et al. (2020) (see also Langer et al. 2020) and is illustrated in the HR diagram in Figure A.2.

The subsequent evolution was modelled using MESA v15140 (see Paxton et al. 2019, and references therein). The input parameters and results for these computations are publicly available. Here we summarise some of the critical assumptions (where these deviate from the default MESA options). Throughout our calculations, we used the type 2 opacity tables of MESA. Following Wang et al. (2020), we modelled convection using standard mixing-length theory, setting the mixing length parameter to $a_{\mathrm{mlt}} \equiv l / H_{\mathrm{p}}=1.5$, where $H_{\mathrm{p}}$ is the local pressure scale height. Convective stability was evaluated using the Ledoux criterion, while we also considered the effects of convective core overshooting (modelled as a step function with $a_{\mathrm{OV}}=0.335$ ), thermohaline mixing $\left(a_{\mathrm{th}}=1.0\right)$ and semi-convection $\left(a_{\mathrm{sc}}=0.01\right.$. Finally, we took into account rotational mixing employing the same parameters as Wang et al. (2020). Mass loss due to stellar winds was modelled using the 'Vink' recipes (Vink et al. 2001; Vink 2017).

We started by evolving a $54.94 M_{\odot}$ star at an SMC metallicity from pre-MS to ZAMS. The ZAMS model was then placed in a $10 \mathrm{AU}, e=0.95$ orbit around a $2.7 M_{\odot}$ pointmass companion. Here, we adopted the default MESA options and parameters to simultaneously solve for the stellar structure and orbital evolution, taking mass loss due to Roche-lobe overflow into account (Paxton et al. 2015). The evolution of the eccentricity was inferred using the relations of Zahn (1977) for radiative envelopes. Once mass transfer became unstable $\left(\dot{M}>0.1 M_{\odot} \mathrm{yr}^{-1}\right)$, the hydrogen-rich envelope was removed using a constant wind of $\left(\dot{M}=0.1 M_{\odot} \mathrm{yr}^{-1}\right)$. The remaining $20.37 M_{\odot} \mathrm{CHeB}$ helium-rich star was then left to evolve until the onset of silicon burning, neglecting further binary interactions.

The evolution of the system from ZAMS until the final merger of the two compact objects is summarised in Figure 2. Figure A.2 illustrates the evolution of the Wang et al. (2020) model on the HR diagram (from ZAMS until the formation of the first compact object).

\section{Dynamical effects of SNe, and post-SN orbital evolution}

The effects of natal SN kicks on the binary orbit were calculated using the standard prescriptions of Hills (1983) and Tauris et al. (2017), which are summarised here for convenience.

The relation between the pre- and post-SN semimajor axes is given by the following:
$\frac{a_{\mathrm{f}}}{a_{\mathrm{i}}}=\left[\frac{1-\Delta M / M}{1-2 \Delta M / M-\left(w / v_{\mathrm{rel}}\right)^{2}-2 \cos \theta\left(w / v_{\mathrm{rel}}\right)}\right]$,

where $\Delta M$ is the amount of mass ejected instantaneously ( $P_{\text {orb }}$ ) during the $\mathrm{SN}, M$ is the total mass of the pre-SN system, $v_{\text {rel }}$ is the relative velocity between the two stars at the time of explosion (assumed to be equal to the relative orbital velocity, $\sqrt{G M / a_{\mathrm{i}}}$, for $\left.e=0\right), w$ is the magnitude of the kick velocity, and $\theta$ is the angle between the kick velocity vector and the pre$\mathrm{SN}$ orbital plane vector. The post-SN eccentricity is given by the following:

$e=\sqrt{1+\frac{2 E_{\mathrm{orb}, \mathrm{f}} L_{o r b, f}^{2}}{\mu_{\mathrm{f}} G^{2} M_{\mathrm{f}, 1}^{2} M_{\mathrm{f}, 2}^{2}}}$

where $L_{\text {orb,f }}=a_{\mathrm{i}} \mu_{\mathrm{f}} \sqrt{\left(v_{\text {rel }}+w \cos \theta\right)^{2}+(w \sin \theta \sin \phi)^{2}}$, with $\phi$ being the kick angle on the plane perpendicular to the pre$\mathrm{SN}$ orbit, $\mu_{\mathrm{f}}$ is the post-SN reduced mass, and $E_{\text {orb,f }}=$ $-G M_{f, 1}^{2} M_{f, 2}^{2} / 2 a_{\mathrm{f}}$ is the post-SN orbital energy. The probability for the system to remain bound is as follows:

$P_{\text {bound }}=\frac{1}{2}\left[1+\left[\frac{1-2 \Delta M / M-\left(w / v_{\text {rel }}\right)^{2}}{2\left(w / v_{\text {rel }}\right)}\right]\right]$.

Finally, a given post-SN system is assumed to survive only if the semi-minor axis is larger than the Roche-lobe radius of the secondary,

$\lambda \equiv a\left(1-e^{2}\right) \leq R_{\mathrm{RL}, 2}$.

Figure A.3 illustrates simulated post-SN systems using the equations above and assuming a kick of a fixed magnitude applied in a random uniform direction. The black-dashed line shows the combination of orbital parameters for which the time to merge equals the Hubble time (13.8 Gyr). The latter was evalulated numerically following Peters (1964) (see also Tauris et al. 2017):

$$
\begin{aligned}
\tau_{\text {merge }}\left(a_{0}, e_{0}\right)=\frac{12}{19} \frac{C_{0}^{4}}{\beta} & \\
& \times \int_{0}^{e_{0}} \frac{e^{29 / 19}\left[1+(121 / 304) e^{2}\right]^{1181 / 2299}}{\left(1-e^{2}\right)^{3 / 2}} d e,
\end{aligned}
$$

where

$C_{0}=\frac{a^{0}\left(1-e_{0}^{2}\right)}{e^{(12 / 19)_{0}}}\left[1+(121 / 304) e_{0}^{2}\right]^{-870 / 2299}$

and

$\beta=\frac{64 G^{3}}{5 c^{5}} M^{2} \mu$

Figure A.4 shows the corresponding $\tau_{\text {merge }}$ distributions for the aforementioned simulations. 

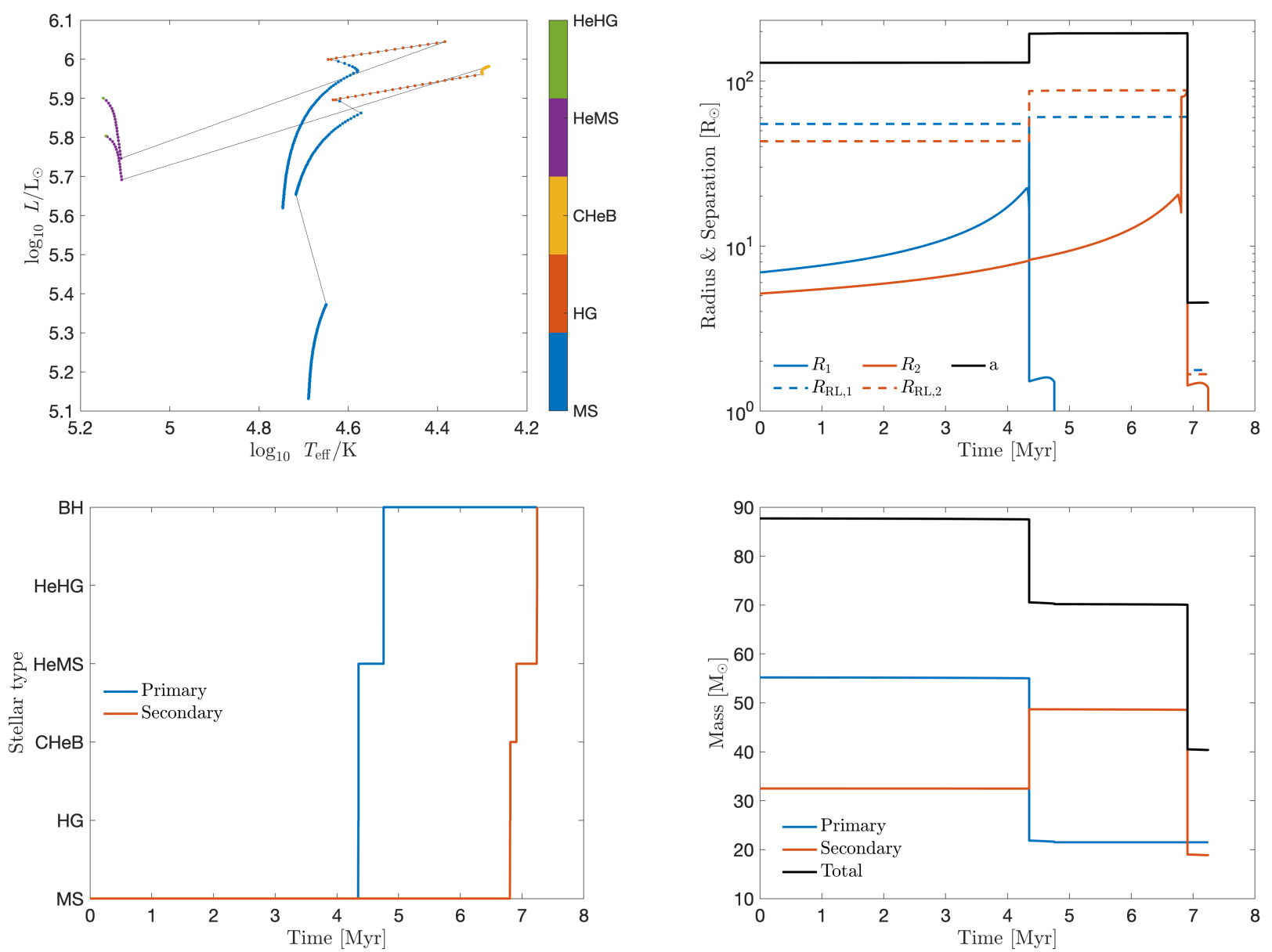

Fig. A.1. Summary of the evolution of the Channel A progenitor system discussed in the main text. The initial binary consists of a $55.2+32.5 M_{\odot}$ ZAMS pair, separated by $0.6 \mathrm{AU}$. The evolution of the system was calculated with the BPS code COMPAS. (Abbreviations: MS: main sequence; HG: Hertzsprung gap; $\mathrm{CHeB}$ : core helium burning; HeMS: helium main sequence; HeHG: Helium Hertzsprung gap; $\mathrm{BH}$ : black hole, $R_{i}$ : radius of the $i$ th star; $R_{\mathrm{RL}, i}$ : Roche-lobe radius of the $i$ th star, $a$ : orbital separation). 


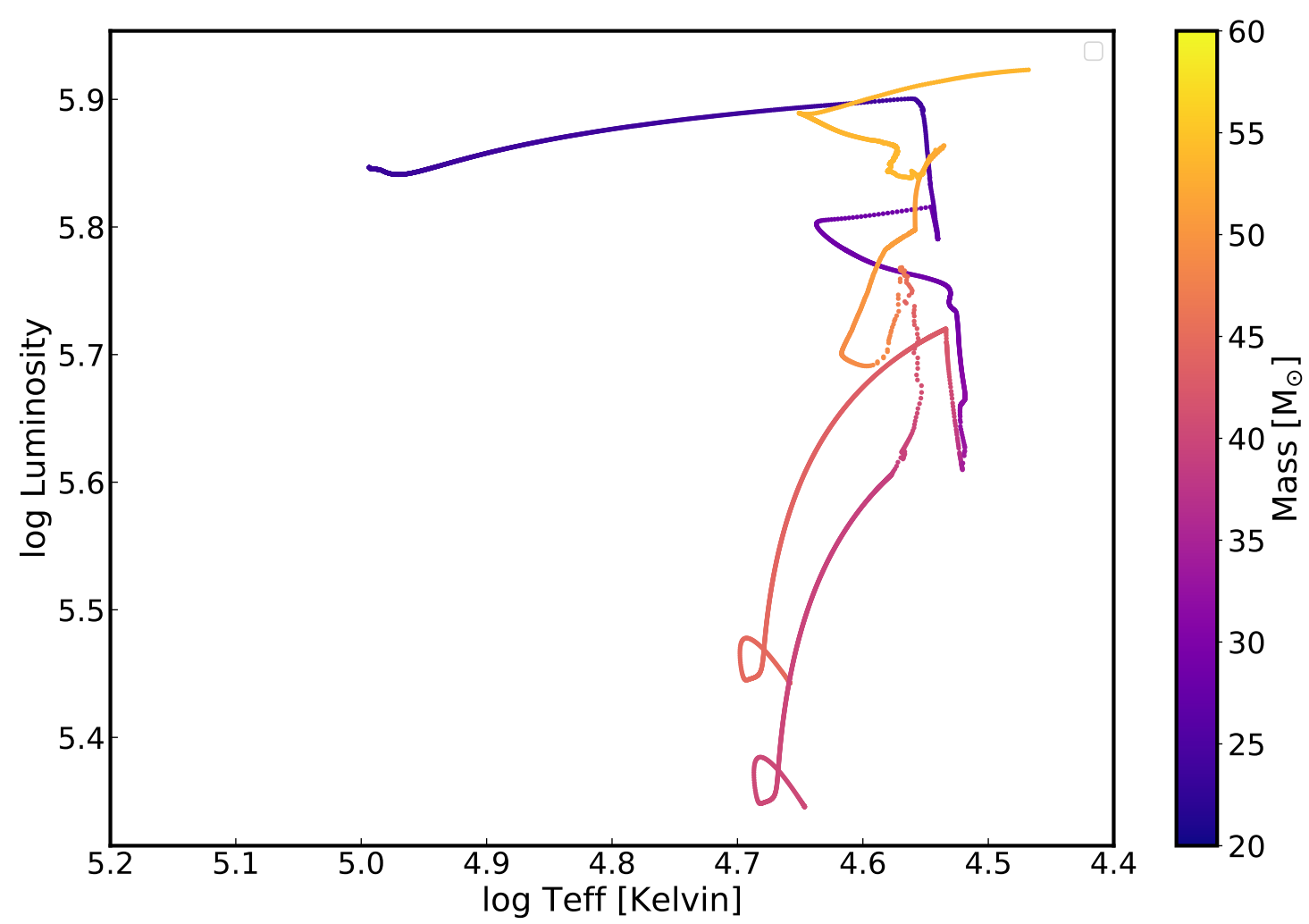

Fig. A.2. Evolution of our Channel B progenitor on the HR diagram from ZAMS until the formation of the first compact object (see text for details)

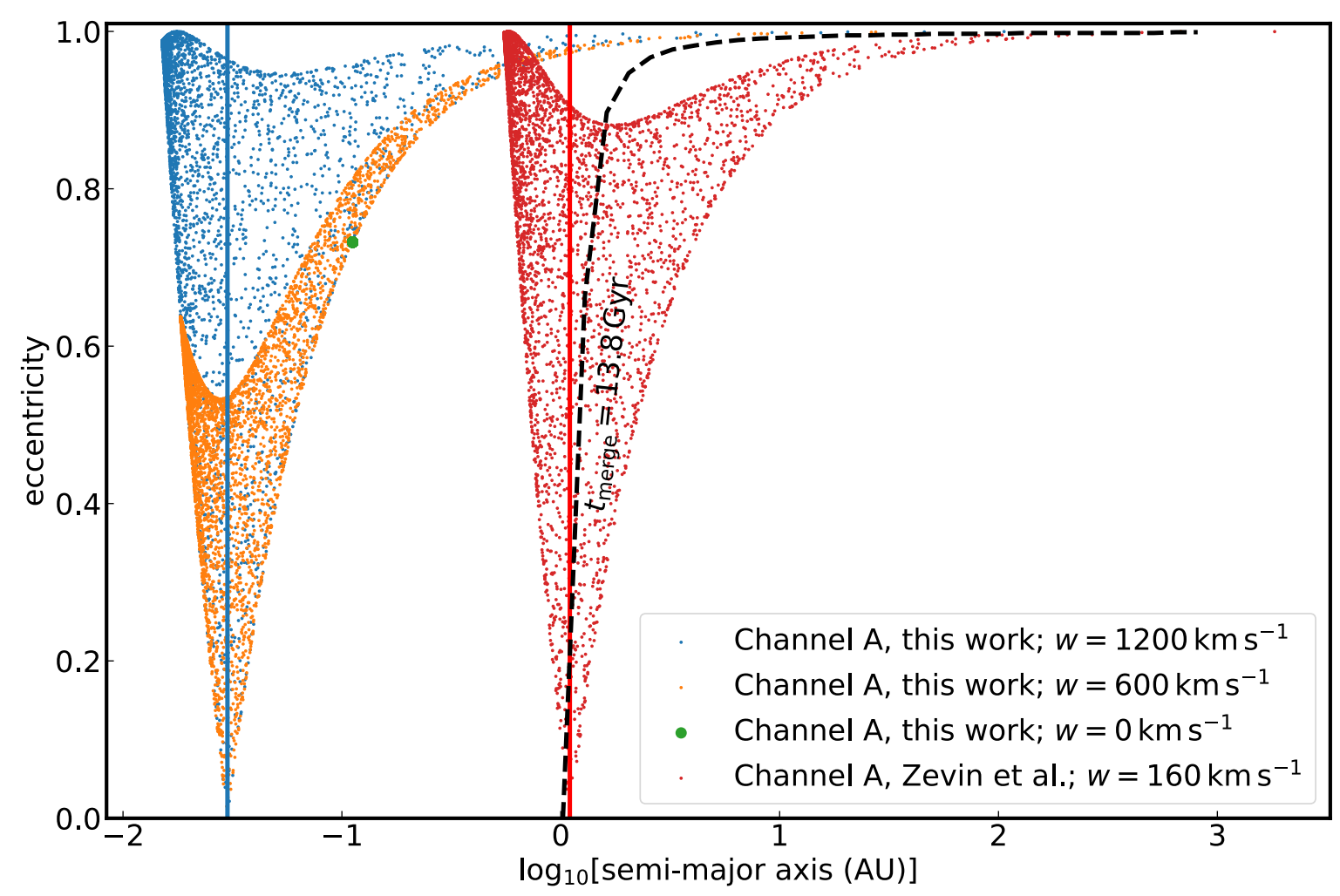

Fig. A.3. Influence of natal SN kicks on the post-SN binary configuration. The scatter plots illustrate the results of a Monte-Carlo simulation for the post-SN distribution of orbital configurations, assuming various initial configurations. Each colour represents a given pre-SN orbit, receiving a kick of fixed amplitude. The kick angles are drawn from uniform distributions (see text and Tauris et al. 2017). Blue, orange, and green points represent the Channel A example discussed in the main text when the second LMG BH is formed. Red points show simulations for the Channel A progenitor discussed in Zevin et al. (2020). Blue and red vertical lines indicate the initial orbital separations of the afforementioned systems. Binaries to the left of the black-dotted line merge within a Hubble time. 


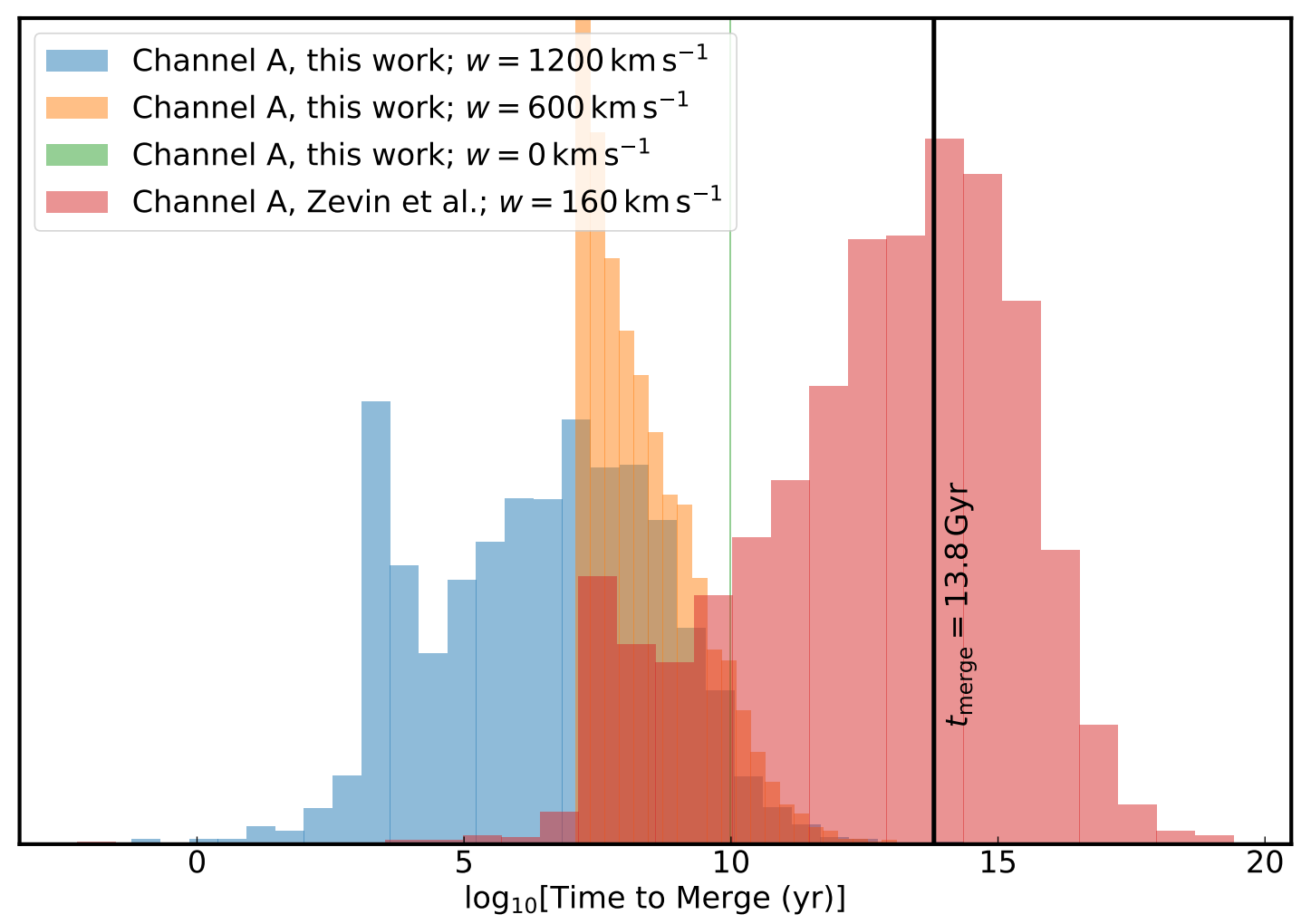

Fig. A.4. Distribution of merge timescales for the binary configurations shown in Fig. A.3 and discussed in the main text. 